\title{
Wind Derivatives: Modeling and Pricing
}

\author{
A. Alexandridis ${ }^{1,2}$, A. Zapranis ${ }^{3}$, \\ ${ }^{2}$ School of Mathematics, Statistics and Actuarial Science. University of Kent. P.O. \\ CT2 7NF, Canterbury, UK. \\ ${ }^{3}$ Department of Accounting and Finance, University of Macedonia of Economics and \\ Social Studies, 156 Egnatia St., P.O. 54006, Thessaloniki, Greece.
}

A.Alexandridis@kent.ac.uk, zapranis@uom.gr

\begin{abstract}
Wind is considered to be a free, renewable and environmentally friendly source of energy. However, wind farms are exposed to excessive weather risk since the power production depends on the wind speed, the wind direction and the wind duration. This risk can be successfully hedged using a financial instrument called weather derivatives. In this study the dynamics of the wind generating process are modeled using a non-parametric non-linear wavelet network. Our model is validated in New York. The proposed methodology is compared against alternative methods, proposed in prior studies. Our results indicate that wavelet networks can model the wind process very well and consequently they constitute an accurate and efficient tool for wind derivatives pricing. Finally, we provide the pricing equations for wind futures written on two indices, the cumulative average wind speed index and the Nordix wind speed index.
\end{abstract}

Keywords: Wind Derivatives, Weather Derivatives, Pricing, Forecasting, Wavelet Networks

\section{Introduction}

Weather derivatives are financial tools that can help organizations or individuals to reduce risk associated with adverse or unexpected weather conditions and can be used as part of a risk management strategy. Weather derivatives linked to various weather indices, such as rainfall, temperature or wind, are extensively traded in Chicago Mercantile Exchange (CME) as well as on Over-The-Counter (OTC) market. According to Challis (1999) and Hanley (1999) nearly \$1 trillion of the US economy is directly exposed to weather risk. It is estimated that nearly $30 \%$ of the US economy and $70 \%$ of the US companies are affected by weather, (CME 2005). The electricity sector is especially sensitive to the temperature and wind since temperature affects the consumption of electricity while wind affects production of electricity in wind farms. Hence, it is logical that energy companies are the main investors of the weather market. In Zapranis and Alexandridis (2008, 2009), Alexandridis (2010) and Zapranis and

\footnotetext{
${ }^{1}$ Corresponding author: $\underline{\text { A.Alexandridis@ @ent.ac.uk }}$
} 
Preprint - Published in Computational Economics, 41 (3), pp. 299-326, 2013.

Alexandridis (2010) a detailed framework for modeling and pricing temperature derivatives was developed. In this study we focus on wind derivatives.

The notional value of the traded wind-linked securities is around \$36 million indicating a large and growing market, (WRMA 2010). However, after the close of the US Future Exchange wind derivatives are traded OTC. The demand from these derivatives exists. However, investors hesitate to enter into wind contracts. The main reasons of the slow growth of the wind market compared to temperature contracts are the difficulty in accurately modeling wind and the challenge to find a reliable model for valuing related contracts. As a result there is a lack of reliable valuation framework that makes financial institutions reluctant to quote prices over these derivatives.

The aim of this paper is to model and price wind derivatives. Wind derivatives are standardized products that depend only on the daily average wind speed measured by a predefined meteorological station over a specified period and can be used by wind (and weather in general) sensitive business such as wind farms, transportation companies, construction companies, theme parks to name few. The financial contracts that are traded are based on the simple daily average wind speed index and this is the reason that we choose to model only the dynamics of the daily average wind speeds. The revenues of each company have a unique dependence and sensitivity to wind speeds. Although wind derivatives and weather derivatives can hedge a significant part of the weather risk of the company always some basis risk will still exists which must be hedged from each company separately. This can be done either by defining a more complex wind index or by taking an additional hedging position. In Cao \& Wei (2003) various examples of how weather derivatives can reduce the basis and volumetric risks in weather sensitive business (ski resorts, restaurants, theme parks, electricity companies) are presented.

Wind is free, renewable and environmentally friendly source of energy, Billinton et al. (1996). Wind derivatives are extensively traded in the electricity sector. While the demand for electricity is closely related to the temperature, the electricity produced by a wind farm is dependent on the wind conditions. The risk exposure of the wind farm depends on the wind speed, the wind direction and in some cases on the wind duration of the wind speed at certain level. However, modern wind turbines include mechanisms that allow turbines to rotate on in the appropriate wind direction, (Caporin and Pres 2010). Wind derivatives can be used as a part of a hedging strategy in wind sensitive 
Preprint - Published in Computational Economics, 41 (3), pp. 299-326, 2013.

business. However, the underlying wind indices do not account for the duration of the wind speed at certain level but rather, usually, measure the average daily wind speed. Hence, the parameter of the duration of the wind speed at certain level is not considered in our daily model. Hence, the risk exposure of a wind sensitive company can be analyzed by quantifying only the wind speed. On the other hand, companies like wind farms that its revenues depend on the duration effect can use an additional hedging strategy that includes this parameter. This can be done by introducing a second index that measures the duration. A similar index for temperature is the Frost Day index.

Many different approaches have been proposed so far for modeling the dynamics of the wind speed process. The most common is the generalized autoregressive moving average (ARMA) approach. There have been a number of studies on the use of linear ARMA models to simulate and forecast wind speed in various locations (Daniel and Chen 1991; Caporin and Pres 2010; Huang and Chalabi 1995; Torres et al. 2005; Billinton et al. 1996; Tol 1997; Kamal and Jafri 1997; Martin et al. 1999; Castino et al. 1998; J. r. a. Benth and Benth 2010). In Kavasseri and Seetharaman (2009) a more sophisticated fractional integrated ARMA (ARFIMA) model was used. Most of these studies did not consider in detail the accuracy of the wind speed forecasts, Huang and Chalabi (1995). On the other hand, Ailliot et al. (2006) apply an autoregressive model (AR) with time-varying coefficients to describe the space-time evolution of wind fields. In F. E. Benth and Saltyte-Benth (2009) a stochastic process, called Continuous AR (CAR) model is introduced in order to model and forecast daily wind speeds. Finally, in Nielsen et al. (2006) various statistical methods were presented for short-term wind speed forecasting. Sfetsos (2002) argues about the use of linear or meteorological models since their prediction error is not significantly lower than the elementary persistent method. Alternatively, some studies use space-state models to simultaneously fit the speed and the direction of the wind, (Castino et al. 1998; Cripps et al. 2005; Martin et al. 1999; Tolman and Booij 1998; Tuller and Brett 1984; Haslett and Raftery 1989).

Alternatively to the linear models, artificial intelligence was applied in wind speed modeling and forecasting. In Sfetsos (2000, 2002), More and Deo (2003), Barbounis et al. (2006), Beyer et al. (1994), Mohandes et al. (1998) and Alexiadis et al. (1998) neural networks were applied in order to model the dynamics of the wind speed process. In 
Preprint - Published in Computational Economics, 41 (3), pp. 299-326, 2013.

Mohandes et al. (2004) support vector machines were used while in Pinson and Kariniotakis (2003) fuzzy neural networks were applied.

Depending on the application, wind modeling is based on hourly, (Yamada 2008; Ailliot et al. 2006; Torres et al. 2005; Sfetsos 2000, 2002; Castino et al. 1998; Martin et al. 1999; Kamal and Jafri 1997; Daniel and Chen 1991), daily, (Caporin and Pres 2010; F. E. Benth and Saltyte-Benth 2009; More and Deo 2003; Tol 1997; Billinton et al. 1996; Huang and Chalabi 1995), weekly or monthly basis, (More and Deo 2003). When the objective is to hedge against electricity demand and production, hourly modeling is used while for weather derivative pricing the daily method is used. More rarely, weekly or monthly modeling is used in order to estimate monthly wind indexes. Since, we want to focus on weather derivative pricing the daily modeling approach is followed; however, the proposed method can be easily adapted in hourly modeling too. Wind speed modeling is much more complicated than temperature modeling since wind has a direction and is greatly affected by the surrounding terrain such as building, trees, etc. (Jewson et al. 2005). However, in Benth and Saltyte-Benth (2009) it is shown that wind speeds dynamics share a lot of common characteristics with the dynamics of temperature derivatives as it was found on Benth and Saltyte-Benth (2007), Zapranis and Alexandridis (2008, 2009), Alexandridis (2010) and Zapranis and Alexandridis (2010). In this context we use a mean reverting Ornstein-Uhlenbeck stochastic process to model the dynamics of the wind speed dynamics were the innovations are driven by a Brownian motion. The statistical analysis reveals seasonality in the mean and variance. In addition we use a novel approach to model the autocorrelation of the wind speeds. More precisely, a wavelet network (WN) is applied in order to capture accurately the autoregressive characteristics of the wind speeds.

The evaluation of the proposed methodology against alternative modeling procedures proposed in prior studies indicates that WNs can accurately model and forecast the dynamics and the evolution of the speed of the wind. The performance of each method was evaluated in-sample as well as out-of-sample and for different time periods.

The rest of the paper is organized as follows. In section 2 a statistical analysis of the wind speed dynamics is presented. In section 3 a linear model is fitted to the data while in section 4 a nonlinear nonparametric $\mathrm{WN}$ is applied. The evaluation of the studied models is presented in section 5. In section 6 we derive the pricing formulas for futures derivatives written on the wind indices. More precisely, in section 6.1 the market price 
Preprint - Published in Computational Economics, 41 (3), pp. 299-326, 2013.

of wind risk is discussed. In section 6.2 and section 6.3 the pricing formulas of futures written on the cumulative average wind speed index and on the Nordix wind index are presented respectively. Finally, in section 7 we conclude.

\section{Modelling the Daily Average Wind Speed}

In this section we derive empirically the characteristics of the daily average wind speed (DAWS) dynamics in New York, USA. The data were collected from NOAA ${ }^{2}$ and correspond to DAWSs. The wind speeds are measured in 0.1 Knots. The measurement period is between $1^{\text {st }}$ of January 1988 and $28^{\text {th }}$ of February 2008. The first 20 years are used for the estimation of the parameters while the remaining two months are used for the evaluation of the performance of the proposed model. In order for each year to have the same number of observations the $29^{\text {th }}$ of February is removed from the data resulting to 7,359 data points. The time-series is complete without any missing values.

In the Fig. 1 the DAWSs for the first 20 years are presented. The descriptive statistics of the in-sample data are presented in Table 1. The values of the data are always positive and range from 1.8 to 32.8 with mean around 9.91. Also, a closer inspection of Fig. 1 reveals seasonality.

The descriptive statistics of the DAWSs indicate that there is a strong positive kurtosis and skewness while the normality hypothesis is rejected based on the Jarque-Bera statistic. The same conclusions can be reached observing the first part of Fig. 2 where the histogram of the DAWSs is represented. The distribution of DAWSs deviates significantly from the normal and it is not symmetrical. In literature the Weibull or the Rayleigh (which is a special case of the Weibull) distributions were proposed in order describe the distribution of the wind speed, ( Brown et al. 1984; Daniel and Chen 1991; Garcia et al. 1998; Justus et al. 1978; Kavak Akpinar and Akpinar 2005; Nfaoui et al. 1996; Torres et al. 2005; Celik 2004; Tuller and Brett 1984). In addition, some studies propose the use of the lognormal distribution, (Garcia et al. 1998), or the Chi-square, (Dorvlo 2002). Finally, in Jaramillo and Borja (2004) a bimodal Weibull and Weibull distribution is used. However, empirical studies favor the use of the Weibull distribution, (Celik 2004; Tuller and Brett 1984).

\footnotetext{
${ }^{2}$ http://www.noaa.gov/
} 
A closer inspection of part (a) of Fig. 2 reveals that the DAWSs in New York follow a Weibull distribution with scale parameter $\lambda=11.07$ and shape parameter $k=3.04$. Following (Brown et al. 1984; Daniel and Chen 1991) in order to symmetrize the data, the Box-Cox transform is applied. The Box-Cox transformation is given by:

$$
W^{(l)}= \begin{cases}\frac{W^{l}-1}{l} & l \neq 0 \\ \ln (W) & l=0\end{cases}
$$

where $W^{(l)}$ is the transformed data. The parameter $l$ is estimated by maximizing the log-likelihood function. Note that the Log-transform is a special case of the Box-Cox transform with $l=0$. The optimal $l$ of the Box-Cox transform for the DAWS in New York is estimated to be 0.014. In the second part of Fig. 2 the histogram of the transformed data can be found while the second row of Table 1 shows the descriptive statistics of the transformed data.

The DAWSs exhibit a clear seasonal pattern which is preserved in the transformed data. The same conclusion can be reached by examining the autocorrelation function (ACF) of the DAWS in the first part of Fig. 3. The seasonal effects are modeled by a truncated Fourier series given by:

$$
S(t)=a_{0}+b_{0} t+\sum_{i=1}^{I_{1}} a_{i} \sin \left(2 \pi i\left(t-f_{i}\right) / 365\right)+\sum_{j=1}^{J_{1}} b_{i} \sin \left(2 \pi j\left(t-g_{j}\right) / 365\right)
$$

In addition we examine the data for a linear trend representing the global warming or the urbanization around the meteorological station. First, we quantify the trend by fitting a linear regression to the DAWS data. The regression is statistically significant with intercept $a_{0}=2.3632$ and slope $b_{0}=-0.000024$ indicating a slightly decrease in the DAWSs. Next, the seasonal periodicities are removed from the detrended data. The remaining statistically significant estimated parameters of equation (2) with $I_{1}=J_{1}=1$ are presented in Table 2. As it is shown on the second part of Fig. 3 the seasonal mean was successfully removed. The same conclusion was reached in previous studies for daily models for both temperature and wind, (Alexandridis 2010; Zapranis and Alexandridis 2008, 2009, 2010; Benth and Saltyte-Benth 2009) 
Preprint - Published in Computational Economics, 41 (3), pp. 299-326, 2013.

\section{The linear ARMA model}

In literature, various methods for studying the statistical characteristics of the wind speed, in daily or hourly measurements, were proposed. However the majority of the studies utilize variations of the general ARMA model, (Ailliot et al. 2006; Billinton et al. 1996; Brett and Tuller 1991; Daniel and Chen 1991; Huang and Chalabi 1995; Kamal and Jafri 1997; Lei et al. 2009; Nfaoui et al. 1996; Rehman and Halawani 1994; Torres et al. 2005). In this paper we will first estimate the dynamics of the detrended and deseasonalized DAWSs process using a general ARMA model and then we will compare our results with a WN.

We define the detrended and deseasonalized DAWS as:

$$
\tilde{W}^{(l)}(t)=W^{(l)}(t)-S(t)
$$

The dynamics of $\tilde{\mathbf{W}}^{(l)}(t)$ are modeled by a vectorial Ornstein-Uhlenbeck stochastic process

$$
d \tilde{W}^{(l)}(t)=\mathbf{a} \tilde{W}^{(l)}(t) d t-I_{p} \sigma(t) d B_{t}
$$

where $I_{p}$ is the $p^{\prime}$ th unit vector in $\mathrm{R}^{p}$ and $\sigma(t)$ is the standard deviation.

First, in order to select the correct ARMA model, we examine the ACF of the detrended and deseasonalized DAWS. A closer inspection of the second part of Fig. 3 reveals that the 1st, 2nd and the 4th lags are significant. On the other hand by examining the Partial Autocorrelation Function (PACF) in Fig. 4 we conclude that the first 4 lags are necessary to model the autoregressive effects of the winds speed dynamics.

In order to find the correct model we estimate the Log Likelihood function (LLF) and the Akaike Information Criterion (AIC). Consistent with the PACF, both criteria suggest that an $\mathrm{AR}(4)$ model is adequate for modeling the wind process since they were minimized when a model with 4 lags was used. The estimated parameters and the corresponding $p$-values are presented in Table 3. It is clear that the three first parameters are statistically very significant since their $p$-value is less than 0.05 . The parameter of the 4th lag is statistically significant with $p$-value 0.0657 . The AIC for this model is 0.46852 while the LLF is -1705.14 .

Observing the residuals of the AR model in the first part of Fig. 5 we conclude that the autocorrelation was successfully removed. However, the ACF of the squared residuals indicates a strong seasonal effect in the variance of the wind speed as it is shown in Fig. 
6. A similar behavior was observed in the residuals of temperature and wind in various studies (Zapranis and Alexandridis 2008, 2009, 2010; Benth and Saltyte-Benth 2009). Following Alexandridis (2010) we model the seasonal variance with a truncated Fourier series:

$$
\sigma^{2}(t)=c_{0}+\sum_{i=1}^{I_{2}} c_{i} \sin (2 i \pi t / 365)+\sum_{j=1}^{J_{2}} d_{j} \sin (2 \pi j t / 365)
$$

Note that we assume that the seasonal variance is periodic and repeated every year, i.e. $\sigma^{2}(t+365)=\sigma^{2}(t)$ where $t=1, \ldots, 7359$. The empirical and the fitted seasonal variance are presented in Fig. 7 while in Table 4 the estimated parameters of equation (5) are presented. Non-surprisingly, the variance exhibits the same characteristics as in the case of temperature. More precisely the seasonal variance is higher in the winter and early summer while it reaches its lower values during the summer period.

Finally, the descriptive statistics of the final residuals are examined. A closer inspection of Table 5 shows that the autocorrelation has successfully removed as indicated by the Ljung-Box Q-statistic. In addition the distribution of the residuals is very close to the normal distribution as it is shown on the first part of Fig. 8. However, small negative skewness exists. More precisely, the residuals have mean 0 and standard deviation of 1. In addition, the kurtosis is 3.03 and the skewness is -0.09 .

Concluding, the previous analysis indicates that an AR(4) model provides a good fit for the wind process while the final residuals are very close to the normal distribution.

\section{Wavelet Networks for Wind Speed Modeling}

In this section WNs are used in the transformed, detrended and deseasonalized wind speed data in order to model the daily dynamics of wind speeds in New York. Motivated by the waveform of the data we expect a wavelet function to better fit the wind speed. In addition, it is expected that the non-linear form of the $\mathrm{WN}$ will provide more accurate representation of the dynamics of the wind speed process both in-sample and out-ofsample.

In the context of the linear ARMA model the mean reversion parameter $a$ is typically assumed to be constant over time. In (Brody et al. 2002) it was mentioned that in general 
$a$ should be a function of time, but no evidence was presented. The impact of a false specification of $a$, on the accuracy of the pricing of temperature derivatives is significant, (Alaton et al. 2002).

In this section, we address that issue, by using a $\mathrm{WN}$ to estimate non-parametrically relationship (4) and then estimate $\mathbf{a}$ as a function of time. In addition, we propose a novel approach for selecting the number of lags in the temperature process first presented by (Alexandridis 2010). Hence a series of speed of mean reversion parameters, $a_{i}(t)$, are estimated. By computing the derivative of the network output with respect to (w.r.t.) the network input we obtain a series of daily values for $a_{i}(t)$. This is done for the first time, and it gives us a much better insight in DAWS dynamics and in wind derivative pricing. As we will see the daily variation of $a_{i}(t)$ is quite significant after all. In addition it is expected that the waveform of the $\mathrm{WN}$ will provide a better fit to the DATs that are governed by seasonalities and periodicities.

Here we present only the basic notion of WNs. For an analytical study on WNs refer to (Alexandridis 2010).

In Fig. 9 the structure and the mathematical expressions of a WN are presented. Given an input vector $\mathbf{x}$ (the harmonics) and a set of weights $\mathbf{w}$ (parameters), the network response (output) $g_{\lambda}(\mathbf{x} ; \mathbf{w})$ is:

$$
g_{\lambda}(\mathbf{x} ; \mathbf{w})=w_{\lambda+1}^{[2]}+\sum_{j=1}^{\lambda} w_{j}^{[2]} \cdot \Psi_{j}(\mathbf{x})+\sum_{i=1}^{m} w_{i}^{[0]} \cdot x_{i}
$$

In that expression, $\Psi_{j}(\mathbf{x})$ is a multidimensional wavelet which is constructed by the product of $m$ scalar wavelets, $\mathbf{x}$ is the input vector, $m$ is the number of network inputs, $\lambda$ is the number of HUs and $w$ stands for a network weight. The multidimensional wavelets are computed by:

$$
\Psi_{j}(x)=\prod_{i=1}^{m} \psi\left(z_{i j}\right)
$$

where

$$
z_{i j}=\frac{x_{i}-w_{(\xi) i j}^{[1]}}{w_{(\zeta) i j}^{[1]}}
$$

The mother wavelet is given by the Mexican Hat function: 
Preprint - Published in Computational Economics, 41 (3), pp. 299-326, 2013.

$$
\psi(\alpha)=\left(1-\alpha^{2}\right) e^{-\frac{1}{2} \alpha^{2}}
$$

Using WNs the generalized version of the autoregressive dynamics of detrended and deseasonalized DAWS estimated nonlinearly and non-parametrically are given by:

$$
\tilde{W}^{(l)}(t+1)=\phi\left(\tilde{W}^{(l)}(t), \tilde{W}^{(l)}(t-1), \ldots\right)+e(t)
$$

where

$$
e(t)=\sigma(t) \varepsilon(t)
$$

and $\tilde{W}^{(l)}(t)$ is given by (3) .

Model (10) uses past DAWS (detrended and deseasonalized) over one period. Using more lags we expect to overcome the strong correlation found in the residuals in models such as in Alaton et al. (2002), Benth and Saltyte-Benth (2007) and Zapranis and Alexandridis (2008). However, the length of the lag series must be selected. In Alexandridis (2010) detailed explanation of how to use WNs in model identification problems is described. Model identification can be separated in two parts, in model selection and in variable significance testing. Since WNs are nonlinear tools, criteria like AIC or LLF cannot be used. Hence, in this section WNs will be used in order to select the significant lags, to select the appropriate network structure, to train a WN in order to learn the dynamics of the wind speeds and, finally, to forecast the future evolution of the wind speeds.

The algorithm developed by Alexandridis (2010) simultaneously estimates the correct number of lags that must be used in order to model the wind speed dynamics and the architecture of the $\mathrm{WN}$ by using a recurrent algorithm. An illustration of the model identification algorithm is presented in Fig. 10. In Alexandridis (2010) we give a concise treatment of the WN theory. Here the emphasis is in presenting the basic notions of the model selection algorithm. For a more detailed exposition on the mathematical aspects of WN we refer to Alexandridis (2010).

Our backward elimination algorithm examines the contribution of each available explanatory variable to the predictive power of the WN. First, the prediction risk of the $\mathrm{WN}$ is estimated as well as the statistical significance of each variable. If a variable is statistically insignificant it is removed from the training set and the prediction risk and the new statistical measures are estimated. The algorithm stops if all explanatory variables are significant. Hence, in each step of our algorithm, the variable with the 
Preprint - Published in Computational Economics, 41 (3), pp. 299-326, 2013.

larger $p$-value greater than 0.1 will be removed from the training set of our model. After each variable removal, a new architecture of the WN will be selected and a new WN will be trained. However the correctness of the decision of removing a variable must be examined. This can be done either by examining the prediction risk or the $\bar{R}^{2}$. If the new prediction risk is smaller than the new prediction risk multiplied by a threshold then the decision of removing the variable was correct. If the prediction risk increased more than the allowed threshold then the variable was reintroduced back to the model. We set this threshold at 5\%. In this study the selected statistical measure is the Sensitivity Based Pruning (SBP) proposed by Moody and Utans (1992). Previous analysis in Alexandridis (2010) indicates that the SBP fitness criterion was found to significantly outperform alternative criteria in the variable selection algorithm. The SBP quantifies the effect on the empirical loss of replacing a variable by its mean. Analytical description of the SBP is given in Alexandridis (2010), Zapranis and Refenes (1999) and Moody and Utans (1992). In each step the SBP and the corresponding $p$ value are calculated. For analytical explanation of each step of the algorithm we refer to Alexandridis (2010).

The proposed variable selection framework will be applied on the transformed, detrended and deseasonalized wind speeds in New York in order to select the length of the lag series. The target values of the WN are the DAWSs. The explanatory variables are lagged versions of the target variable. The relevance of a variable to the model is quantified by the SBP criterion which was introduced in Moody and Utans (1992). Initially the training set contains the dependent variable and 7 lags. The analysis in the previous section indicates that a training set with 7 lags will provide all the necessary information of the ACF of the detrended and deseasonalized DAWSs. Hence, the initial training set consists of 7 inputs, 1 output and 7,293 training pairs.

Table 6 summarizes the results of the model identification algorithm for the New York. Both the model selection and the variable selection algorithms are included in Table 6. The algorithm concluded in 4 steps and the final model contains only 3 variables, i.e 3 lags. The prediction risk for the reduced model is 0.0937 while for the original model was 0.0938 indicating that the predictive power of the $\mathrm{WN}$ was slightly increased. On the other hand the empirical loss slightly increased from 0.0467 for the initial model to 0.0468 for the reduced model indicating that the explained variability (unadjusted) slightly decreased. Finally, the complexity of the network structure and number of 
Preprint - Published in Computational Economics, 41 (3), pp. 299-326, 2013.

parameters were significantly reduced in the final model. The initial model needed 1 hidden unit (HU) and 7 inputs. Hence, 23 parameters were adjusted during the training phase. Hence the ratio of the number of training pairs $n$ to the number of parameters $p$ was 317.4. In the final model only $2 \mathrm{HU}$ and 3 inputs were used. Hence only 18 parameters were adjusted during the training phase and the ratio of the number of training pairs $n$ to the number of parameters $p$ was 405.6.

The proposed algorithm suggests that a WN needs only 3 lags to extract the autocorrelation from the data while the linear model needed 4 lags. A closer inspection of Table 6 reveals that the WN with 3 and 4 lags have the same predictive power insample and out-of-sample. Hence, we chose the simpler model.

The daily values of the speed of mean reversion function $a_{i}(t)$ (7,293 values) are depicted in Fig. 11. Since there are 3 significant lags, there are three mean-reverting functions. Our results indicate that the speed of mean reversion is not constant. On the contrary, its daily variation is quite significant; this fact naturally has an impact on the accuracy of the pricing equations and it has to be taken into account, Alaton et al. (2002). Intuitively, it was expected $a_{i}(t)$ not to be constant. If the wind speed today is away from the seasonal average then it is expected that the speed of mean reversion to be high; i.e. wind speed cannot deviate from its seasonal mean for long periods.

Examining the second part of Fig. 5 we conclude that the autocorrelation was successfully removed from the data; however, the seasonal autocorrelation in the squared residuals is still present as it is shown in Fig. 6. We will remove the seasonal autocorrelation using equation (5). The estimated parameters are presented in Table 7 and as it was expected their values are similar to those of the case of the linear model. In Fig. 7 the empirical and the fitted seasonal variance is presented. The variance is higher at winter period while it reaches its minimum during the summer period.

Finally, examining the final residuals of the WN model, we observe that the distribution of the residuals is very close to the normal distribution as it is shown in Fig. 8 while the autocorrelation was successfully removed from the data. In addition we observe an improvement in the distributional statistics in contrast to the case of the linear model. The distributional statistics of the residuals are presented in Table 8.

Concluding, the distributional statistics of the residuals indicate that in-sample the two models can accurate represent the dynamics of the DAWSs however an improvement is evident when a nonlinear nonparametric $\mathrm{WN}$ is used. 
Preprint - Published in Computational Economics, 41 (3), pp. 299-326, 2013.

\section{Forecasting daily average wind speeds}

In this section the proposed model will be validated out-of-sample. In addition the performance of the model will be tested against two models, first, against the linear model previously described and second, against the simple persistent method usually referred as benchmark. The linear model is the AR(4) model described in the previous section. The persistent method assumes that the today's and tomorrow's DAWSs will be equal, i.e. $W^{*}(t+1)=W(t)$ where the $W^{*}$ indicates the forecasted value.

The three models will be used for forecasting DAWSs for two different periods. Usually wind derivatives are written for a period of a month. Hence, DAWSs for 1 and 2 months will be forecasted. The out-of-sample dataset correspond to the period from January $1^{\text {st }}$ to February $28^{\text {th }} 2008$ and were not used for the estimation of the linear and nonlinear models. Note that our previous analysis reveals that the variance in higher in the winter period indicating that it is more difficult to forecast accurately DAWS for these two months. In order to compare our results, the Monte Carlo approach is followed. We simulate 10.000 paths and calculate the average error criteria.

In Table 9 the performance of the three methods when the forecast window is one month is presented. Various error criteria are estimated like the Mean, Median and Maximum Absolute Error (Max. AE), the Mean Square Error (MSE), the Position of Change in Direction (POCID) and the Independent POCID. As it is shown on Table 9 our proposed method outperforms both the persistent and the AR(4) model. The AR(4) model performs better than the naïve persistent method however all error criteria are further improved when a nonlinear WN is used. The MSE is 16.3848 for the persistent method, 10.5376 for the AR(4) model and 10.4643 for the WN. In addition our model can predict more accurately the movements of the wind speed since the POCID is $80 \%$ for the WN and the AR(4) models while it is only $47 \%$ for the persistent method. Moreover the IPOCID is $37 \%$ for the proposed model while it is only $33 \%$ for the other two methods.

Next, the three forecasting methods are evaluated in two months day-ahead forecasts. The results are similar and presented in Table 10. The proposed WN outperforms the other two methods. Only the Md. AE is slightly better when the AR(4) model is used. However the IPOCID is $38 \%$ for the AR(4) method while it is $43 \%$ for the WN. Also, 
Preprint - Published in Computational Economics, 41 (3), pp. 299-326, 2013.

our results indicate that the benchmark persistent method produces significantly worse forecasts.

Our results indicate that the $\mathrm{WN}$ can forecast the evolution of the dynamics of the DAWSs and hence they constitute an accurate tool for wind derivatives pricing.

In order to have a better insight of the performance of each method the cumulative average wind speed (CAWS) index is calculated. Since we are interested in weather derivatives, one common index is the sum of the daily rainfall index over a specific period. In

Table 11 the estimation of three methods are presented. More precisely, the WN, the $\mathrm{AR}(4)$ as well as the historical burn analysis (HBA) method are compared. The HBA is a simple statistical method that estimates the performance of the index over the specific period the previous years and it is often used in the industry. In other words, is the average of 20 years of the index over the period of January and February and it serves as a benchmark.

The final row of

Table 11 presents the actual values of the cumulative rainfall index. An inspection of Table 11 reveals that the WN significantly outperforms the other two methods. For the first case,

Where forecasts for one month ahead are estimated, the forecast of the CAWS index using $\mathrm{WN}$ is 312.7 while the actual index is 311.2 . On the other hand the forecast using the AR(4) model is 305.1. However, when the forecast period increases the forecast of the AR(4) model significantly deviates. More precisely, for the second case, the forecast of the WN is 591.1 while the actual index is 600.6 and the $\mathrm{AR}(4)$ forecast is 579.5. Finally, we have to mention that the WN use less information than the AR(4) model, since in the case of WN only the information of 3 lags are used.

Since we are interested in wind derivatives and the valuation of wind contracts, next an illustration of the performance of each method using a theoretical contract is presented. A common wind contract has a tick size of 0.1 knots and pays $20 \$$ per tick size. Hence, for the case of an one month contract the AR(4) method underestimates the contract size for $1,200 \$$ while the WN overestimates the contract for $300 \$$ only. Similarly for the case of a two month contract the AR(4) method underestimates the contract size for $4,220 \$$ while the WN underestimates the contract for $1,900 \$$. 
Preprint - Published in Computational Economics, 41 (3), pp. 299-326, 2013.

Incorporating meteorological forecasts can lead to a potentially significant improvement of the performance of the proposed model. Meteorological forecasts can be easily incorporated in both the linear and the WN models previously presented. A similar approach was followed for temperature derivatives by Dorfleitner and Wimmer (2010) for temperature derivatives. However, this method cannot be always applied. Despite great advances in meteorological science, weather still cannot be predicted precisely and consistently and forecasts beyond 10 days are not considered accurate, Wilks (2011). If the day that the contract is traded is during or close to the life of the derivative (during the period that wind measurements are considered) the meteorological forecasts can be incorporated in order to improve the performance of the methods. However, very often weather derivatives are traded long before the start of the life of the derivative. More precisely, very often weather derivatives are traded months or even a season before the starting day of the contract. In this case, meteorological forecasts cannot be used.

\section{Pricing Wind Derivatives}

In this section the pricing formulas for wind derivatives are presented under the assumption of a normal driving noise process. The analysis that performed in the previous section indicates the assumption that the final residuals, after dividing out the seasonal variance, follow a normal distribution is justified.

When the market is complete, a unique risk-neutral probability measure $Q \sim P$ can be obtained, where $P$ is the real world probability measure. This change of measure turns the stochastic process into a martingale. Hence, financial derivatives can be priced under the risk-neutral measure by the discounted expectation of the derivative payoff. The weather market is an incomplete market in the sense that the underlying weather derivative cannot be stored or traded. Moreover the market is relatively illiquid. In principle, (extended) risk-neutral valuation can be still carried out in incomplete markets, (Xu et al. 2008). However, in incomplete markets a unique price cannot be obtained using the no-arbitrage assumption. In other words, many equivalent martingales exist and as a result, only bounds for prices on contingent claims can be provided, (Xu et al. 2008).

The change of measure from the real world to the risk-neutral world under the dynamics of a BM can be performed using the Girsanov theorem. The Girsanov theorem tells us 
how changes in the measure affect a stochastic process. Then the discounted expected payoff of the various weather contracts can be estimated. However, in order to estimate the expected payoff of each derivative, the solution of the stochastic differential equation that describes the wind speed dynamics must be solved. This can be done by applying the Itô's Lemma when a BM is considered.

The statistical analysis indicates that the transformed DAWSs can be modeled by a vectorial mean reverting Ornstein-Uhlenbeck process where the speed of mean reversion variable is a function of time:

$$
d W_{t}^{(l)}=S(t)+\kappa(t)\left(W_{t-1}^{(l)}-S(t-1)\right) d t+I_{p} \sigma(t) d B_{t}
$$

where $S(t)$ is the seasonal function, $\sigma(t)$ is the seasonal variance which is bounded by zero, $\boldsymbol{\kappa}(t)$ is the speed of mean reversion and $B_{t}$ is the driving noise process.

Using the Girsanov's theorem, under the risk neutral measure $Q \sim P$, we have that:

$$
d B_{t}^{\theta}=d B_{t}-\theta(t)
$$

where $\theta(t)$ is the market price of wind risk and

$$
\int_{0}^{T} \theta^{2}(t) d t<\infty
$$

Hence, applying Itô Formula on equations (12) and (13) the solution of the transformed DAWS $\tilde{\mathbf{W}}^{(l)}(t)$ under the risk neutral measure $Q$ is given by:

$$
\begin{aligned}
W_{t}^{(l)} & =S(t)+e^{\int_{0}^{t} \mathbf{\kappa}(z) d z}\left(W_{0}^{(l)}-S(0)\right)+e^{\int_{0}^{t} \boldsymbol{\kappa}(z) d z} \int_{0}^{t} \sigma(s) \theta(s) e^{-\int_{0}^{s} \boldsymbol{\kappa}(z) d z} I_{p} d s \\
& +e^{\int_{0}^{t} \boldsymbol{\kappa}(z) d z} \int_{0}^{t} \sigma(s) e^{-\int_{0}^{s} \boldsymbol{\kappa}(z) d z} I_{p} d B_{t}^{\theta}
\end{aligned}
$$

The proposed model is an extension of the $\operatorname{CAR}(p)$ introduced by (Brockwell and Marquardt 2005) and applied by (F. E. Benth and Saltyte-Benth 2009) in wind derivative pricing. Hence, we follow a similar pricing approach presented in (F. E. Benth and Saltyte-Benth 2009).

The transformed, detrended and deseasonalized DAWS $\tilde{W}_{t}^{(l)}=W_{t}^{(l)}-S(t)$ are normally distributed with mean: 
Preprint - Published in Computational Economics, 41 (3), pp. 299-326, 2013.

$$
\mu_{\theta}\left(t, s, \tilde{W}_{t}^{(l)}\right)=I_{p}^{\prime} e^{\int_{t}^{s} \kappa(z) d z} \tilde{W}_{s}^{(l)}+e^{\int_{t}^{s} \kappa(z) d z} \int_{t}^{s}\left\{I_{p}^{\prime} e^{-\int_{t}^{u} \kappa(z) d z} I_{p}\right\} \sigma(u) \theta(u) d u
$$

And variance

$$
\Sigma^{2}(t, s)=e^{2 \int_{t}^{s} \boldsymbol{\kappa}(z) d z} \int_{t}^{s} \sigma^{2}(s)\left\{I_{p}^{\prime} e^{-2 \int_{t}^{u} \mathbf{\kappa}(z) d z} I_{p}\right\} d u
$$

\subsection{The market price of wind risk}

The wind index is not a tradeable asset, hence the wind derivatives market is a classical incomplete market. In incomplete markets, the derivative contract cannot be replicated by the underlying index. Moreover, in incomplete markets there are infinite equivalent martingale measures. Here, we limit our choices by selecting a parametric class of probabilities $Q^{\theta}$, where $\theta(t)$ is square integrable function as it is assumed by equation (14)

The parameter $\theta(t)$ is called the market price of wind risk. The choice of $\theta(t)$ actually captures the risk preference of the market participants. In other words, it reflects the trader's view on exposing themselves to risk, Benth (2004). The parameter $\theta(t)$ was introduced in our model by applying the Girsanov's theorem. The change of measure of an asset's stochastic process is closely related to the concept of the market price of risk, Xu et al. (2008). Actually the drift rate of the asset's stochastic process is corrected by a parameter that reflects the market price of risk, Xu et al. (2008).

In most studies so far the market price of risk in the weather market was considered zero. However, recently many studies examine the market price of risk and found that it is different than zero.

The most common approach in estimating the market price of risk is the one presented in Alaton et al. (2002) and it was followed by Bellini (2005), Benth et al. (2009) and Hardle and Lopez Cabrera (2011). Alaton et al. (2002) suggest that the market price of risk can be estimated from the market data. More precisely the market price of risk is derived as follows: we examine what value of the $\theta(t)$ gives a price from the theoretical model that fits the observable market price. 
Preprint - Published in Computational Economics, 41 (3), pp. 299-326, 2013.

In Hardle and Lopez Cabrera (2011), the implied market price of risk from Berlin was estimated. Their results indicate that the market price of risk for CAT derivatives is different from zero and shows a seasonal structure that increases as the expiration date of the temperature future increases. Alternatively, Turvey (2005) proposed to estimate the market price of risk by using the capital asset pricing model. Cao and Wei (2004) and Richards et al. (2004) apply a generalized Lucas' (1978) equilibrium pricing model to study the market price of risk. In that framework direct estimation of the weather risk's market price is avoided, Xu et al. (2008). Their findings indicate that the market price of risk associated with the temperature variable is significant. They also conclude that the market price of risk affects option values much more than forward prices, mainly due to the payoff specification.

The market price of wind risk is necessary in pricing wind derivatives. However, in order to estimate $\theta$ the actual prices of derivatives are required. Since the shutdown of the US Future Exchange wind derivatives are traded only in the OTC market and as a result it is hard to obtain market data. Hence, it is very difficult to estimate the market price of wind risk. However, the trading volume of wind derivatives is increasing every year, (WRMA 2010) and it is expected that wind derivatives will be soon included in the listed products of the CME.

A solution to this problem is presented by Benth et al. (2009) where they study the market price of risk for temperature derivatives in various Asian cities. The market price of risk was estimated by calibrating model prices. Their results indicate that the market price of risk for Asian temperature derivatives is different from zero and shows a seasonal structure that comes from the seasonal variance of the temperature process. Their empirical findings suggest that by knowing the formal dependence of the market price of risk on seasonal variation, one can infer the market price of risk for regions where weather derivative market does not exist.

Similar, in Huang et al. (2008) a pricing method for temperature derivatives in Taiwan is presented. Since, no active weather market exists in Taiwan the parameter $\theta$ is approximated by a function of the market price of risk of the Taiwan Stock Exchange. As it is already mentioned, the market price of risk is connected with the risk preference of the market participants. Alternatively, utility functions that describe the risk reference of the market preference can be applied. For example in Xu et al. (2008) an indifference pricing approach which is based on utility maximization is proposed. 


\subsection{The cumulative average wind speed index}

In this section we derive the pricing equation for the Cumulative Average Wind Speed Index (CAWS). The CAWS index is the sum of the DAWSs over a specific period $\left[\tau_{1}, \tau_{2}\right]$ and it is given by:

$$
C A W S=\int_{\tau_{1}}^{\tau_{2}} W(s) d s
$$

Our aim is to give a mathematical expression for the CAWS futures prices. If $Q$ is the risk-neutral probability and $r$ is the constant compounding interest rate, then the arbitrage-free future price of a CAWS contract at time $t \leq \tau_{1}<\tau_{2}$ is given by:

$$
e^{-r\left(\tau_{2}-1\right)} \mathrm{E}_{Q}\left[\int_{\tau_{1}}^{\tau_{2}} W(s) d s-F_{C A W S}\left(t, \tau_{1}, \tau_{2}\right) \mid \mathcal{F}_{t}\right]=0
$$

and since $F_{\text {CAWS }}$ is $\mathfrak{F}_{t}$ adapted we derive the price of a CAWS futures to be

$$
F_{C A W S}\left(t, \tau_{1}, \tau_{2}\right)=\mathrm{E}_{Q}\left[\int_{\tau_{1}}^{\tau_{2}} W(s) d s \mid \mathcal{F}_{t}\right]
$$

To derive the future price, we must calculate the conditional expectation of $W(s)$ given $\mathfrak{F}_{t}$, for $s \geq t$. This is done in the following Lemma first presented in F. E. Benth and Saltyte-Benth (2009). For reasons of completeness we reproduce this Lemma here.

Lemma 1. Let $0 \leq t \leq s \leq T$, then for $l \in[0,1]$ it holds that

$$
\mathrm{E}_{Q}\left[W(s) \mid \mathcal{F}_{t}\right]=M_{1 / l}\left(1+l\left(S(s)+\mu_{\theta}\left(t, s, \tilde{W}_{t}^{(l)}\right), l^{2} \Sigma^{2}(t, s)\right)\right)
$$

where $M_{k}\left(a, b^{2}\right)$ is the $k^{\text {th }}$ moment of a normal random variable with mean $a$ and variance $b^{2}$ and $\mu_{\theta}\left(t, s, \tilde{W}_{t}^{(l)}\right)$ and $\Sigma^{2}(t, s)$ are given by (16) and (17) respectively.

Proof. From equations (1) and (15) we have that the wind speed at time $s$ given $\mathcal{F}_{t}$ can be represented (for $l \neq 0$ ) as

$$
W(s)=\left[l\left(S(s)+\mu_{\theta}\left(t, s, \tilde{W}_{t}^{(l)}\right)+\Sigma(t, s)\right) Z+1\right]^{1 / l}
$$

where $Z$ is a standard normally distributed random variable independent of $\mathfrak{F}_{t}$. Further, $\mu_{\theta}\left(t, s, \tilde{W}_{t}^{(l)}\right)$ is $\mathscr{F}_{t}$-measurable. Hence, the result follows from a direct calculation. The lognormal case $l=0$ follows similarly. 
Preprint - Published in Computational Economics, 41 (3), pp. 299-326, 2013.

Hence, the arbitrage-free price of the CAWS index it easily follows from Lemma 1.

Proposition 1. The arbitrage-free price of CAWS index at time $t \leq \tau_{1}<\tau_{2}$ is given by:

$$
F_{C A W S}\left(t, \tau_{1}, \tau_{2}\right)=\int_{\tau_{1}}^{\tau_{2}}\left(M_{1 / l}\left(1+l\left(S(s)+\mu_{\theta}\left(t, s, \tilde{W}_{t}^{(l)}\right), l^{2} \Sigma^{2}(t, s)\right)\right)\right) d s
$$

where $M_{k}\left(a, b^{2}\right)$ is the $k^{\text {th }}$ moment of a normal random variable with mean $a$ and variance $b^{2}$ and $\mu_{\theta}\left(t, s, \tilde{W}_{t}^{(l)}\right)$ and $\Sigma^{2}(t, s)$ are given by (16) and (17) respectively.

Proof. We have from (20) that

$$
F_{C A W S}\left(t, \tau_{1}, \tau_{2}\right)=\mathrm{E}_{Q}\left[\int_{\tau_{1}}^{\tau_{2}} W(s) d s \mid \mathcal{F}_{t}\right]
$$

and using Itô's isometry we can interchange the expectation and the integral which results to

$$
\mathrm{E}_{Q}\left[\int_{\tau_{1}}^{\tau_{2}} W(s) d s \mid \mathcal{F}\right]=\int_{\tau_{1}}^{\tau_{2}} \mathrm{E}_{Q}[W(s) \mid \mathscr{F}] d s
$$

and from a direct application from Lemma 1 we have that

$$
F_{C A W S}\left(t, \tau_{1}, \tau_{2}\right)=\int_{\tau_{1}}^{\tau_{2}}\left(M_{1 / l}\left(1+l\left(S(s)+\mu_{\theta}\left(t, s, \tilde{W}_{t}^{(l)}\right), l^{2} \Sigma^{2}(t, s)\right)\right)\right) d s
$$

\subsection{The Nordix wind speed index}

In this section we derive the pricing equations for the Nordix Wind Speed index which is the index that the US Future Exchange used to settle wind derivatives. The Nordix Index is given by:

$$
I\left(\tau_{1}, \tau_{2}\right)=100+\sum_{s=\tau_{1}}^{\tau_{2}}\left(W(s)-w_{20}(s)\right)
$$

and measures the daily wind speed deviations from the mean of the past 20 years over a period $\left[\tau_{1}, \tau_{2}\right]$. 
Preprint - Published in Computational Economics, 41 (3), pp. 299-326, 2013.

Proposition 2. The arbitrage-free price of a Nordix Wind Future Speed index at time $t \leq \tau_{1}<\tau_{2}$ is given by:

$$
\begin{aligned}
& F_{N W I}\left(t, \tau_{1}, \tau_{2}\right)=100-\sum_{s=\tau_{1}}^{\tau_{2}} w_{20}(s) \\
& +\sum_{s=\tau_{1}}^{\tau_{2}} M_{1 / l}\left(1+l\left(S(s)+\mu_{\theta}\left(t, s, \tilde{W}_{t}^{(l)}\right), l^{2} \Sigma^{2}(t, s)\right)\right.
\end{aligned}
$$

where $M_{k}\left(a, b^{2}\right)$ is the $k^{\text {th }}$ moment of a normal random variable with mean a and variance $b^{2}$.

Proof. If $Q$ is the risk-neutral probability and $r$ is the constant compounding interest rate, the arbitrage-free price of Nordix Wind Future index at time $t \leq \tau_{1}<\tau_{2}$ is given by:

$$
e^{-r\left(\tau_{2}-t\right)} \mathrm{E}_{Q}\left[100-\sum_{s=\tau_{1}}^{\tau_{2}} w_{20}(s)+\sum_{s=\tau_{1}}^{\tau_{2}} W(s)-F_{N W I}\left(t, \tau_{1}, \tau_{2}\right) \mid \mathfrak{F}\right]=0
$$

and since $F_{N W I}\left(t, \tau_{1}, \tau_{2}\right)$ is $\mathscr{F}_{t}$ adapted we derive the price of a Nordix Wind Future Index to be:

$$
F_{N W I}\left(t, \tau_{1}, \tau_{2}\right)=100-\sum_{s=\tau_{1}}^{\tau_{2}} w_{20}(s)+\mathrm{E}_{Q}\left[\sum_{s=\tau_{1}}^{\tau_{2}} W(s) \mid \mathcal{F}_{t}\right]
$$

Applying the Lemma 1 we find the explicit solution for the price of the Nordix Wind Future Index:

$$
\begin{aligned}
& F_{N W I}\left(t, \tau_{1}, \tau_{2}\right)=100-\sum_{s=\tau_{1}}^{\tau_{2}} w_{20}(s) \\
& +\sum_{s=\tau_{1}}^{\tau_{2}} M_{1 / l}\left(1+l\left(S(s)+\mu_{\theta}\left(t, s, \tilde{W}_{t}^{(l)}\right), l^{2} \Sigma^{2}(t, s)\right)\right.
\end{aligned}
$$

where $M_{k}\left(a, b^{2}\right)$ is the $k^{\text {th }}$ moment of a normal random variable with mean a and variance $b^{2}$. 
Preprint - Published in Computational Economics, 41 (3), pp. 299-326, 2013.

\section{Conclusions}

In this study DAWSs from New York were studied. Our analysis revealed strong seasonality in the mean and variance. The DAWSs were modeled by a mean reverting Ornstein-Uhlenbeck process in the context of wind derivatives pricing. In this study the dynamics of the wind generating process are modeled using a non-parametric nonlinear WN. Our proposed methodology was compared in-sample and out-of-sample against two methods often used in prior studies. The characteristics of the wind speed process are very similar to the process of daily average temperatures. Our results indicate a slight downward trend and seasonality in the mean and variance. In addition the seasonal variance is higher in the winter while it reaches its lower values during the summer period.

Our method is validated in a two month ahead out of sample forecast period. Moreover, the various error criteria produced by the $\mathrm{WN}$ are compared against the linear AR model and the persistent method. Results show that the WN outperforms the other two methods, indicating that WNs constitute an accurate model for forecasting DAWSs. More precisely the WN's forecasting ability is stronger in both samples. Testing the fitted residuals of the WN we observe that the distribution of the residuals is very close to the normal. Also, the WN needed only the information of the past 3 days while the linear method suggested a model with 4 lags. Finally, we provided the pricing equations for wind futures of the Nordix index. Although we focused on DAWSs our model can be easily adapted in hourly modeling.

Finally, the pricing formulas for wind derivatives are presented under the assumption of a normal driving noise process. More precisely the pricing formulas of futures contracts written on the CAWS index and Nordix wind speed index were presented.

The results in this study are preliminary and can be further analyzed. More precisely alternative methods for estimating the seasonality in the mean and in the variance can be developed. Alternative methods could improve the fitting to the original data as well as the training of the WN. In addition, the inclusion of meteorological forecasts can further improve the forecasting performance of the WNs.

Also, it is important to test the largest forecasting window of each method. Since meteorological forecasts of a window larger than few days are considered inaccurate this analysis will suggest the best model according to the desired forecasting interval. 
Preprint - Published in Computational Economics, 41 (3), pp. 299-326, 2013.

Finally, a large scale comparison must be conducted. Testing the proposed methods as well as more sophisticated models, like general ARFIMA or GARCH, in various meteorological stations will provide a better insight in the dynamics of the DAWS as well as in the predictive ability of each method.

\section{Acknowledgement}

We would like to thank the anonymous referees for the constructive comments that substantially improved the final version of this paper.

\section{References}

Ailliot, P., Monbet, V., \& Prevosto, M. (2006). An autoregressive model with time-varying coefficients for wind fields. Envirometrics, 17, 107-117.

Alaton P, Djehince B, Stillberg D (2002) On Modelling and Pricing Weather Derivatives. Applied Mathematical Finance, 9, 1-20

Alexandridis, A. (2010). Modelling and Pricing Temperature Derivatives Using Wavelet Networks and Wavelet Analysis. University of Macedonia, Thessaloniki, Greece.

Alexiadis, M. C., Dokopoulos, P. S., Sahsamanoglou, H. S., \& Manousaridis, I. M. (1998). Short-term forecasting of wind speed and related electrical power Solar Energy, 63(1), 61-68.

Barbounis, T. G., Theocharis, J. B., Alexiadis, M. C., \& Dokopoulos, P. S. (2006). Long-term wind speed and power forecasting using local recurrent neural network models. Energy Conversion, IEEE Transactions on, 21(1), 273-284.

Bellini F (2005) The weather derivatives market: Modelling and pricing temperature. Ph.D Thesis, University of Lugano, Lugano, Switzerland.

Benth FE (2004) Option theory with stochastic analysis. Springer, Berlin.

Benth, F. E., Hardle, W. K., \& Lopez Cabrera, B. (2009). Pricing of Asian temperature risk. SFB649 Working Paper. Berlin: Humboldt-Universitat zu Berlin.

Benth, F. E., \& Saltyte-Benth, J. (2007). The volatility of temperature and pricing of weather derivatives. Quantitative Finance, 7(5), 553-561.

Benth, F. E., \& Saltyte-Benth, J. (2009). Dynamic Pricing of Wind Futures. Energy Economics, 31, 1624.

Benth, J. r. a., \& Benth, F. E. (2010). Analysis and modelling of wind speed in New York. Journal of Applied Statistics, 37(6), 893 - 909.

Beyer, H. G., Degner, T., Hausmann, J., Hoffmann, M., \& Rujan, P. Short-term prediction of wind speed and power outputof a wind turbine with neural netwroks. In 2nd European Congress on Intelligent Techniques and Soft Computing, Aachen, Germany, 20-23 September 1994

Billinton, R., Chen, H., \& Ghajar, R. (1996). Time-series Models for Reliability Evaluation of Power Systems Including Wind Energy. Microelectronics and Reliability, 36(9), 1253-1261.

Brett, A. C., \& Tuller, S. E. (1991). The Autocorrelation of Hourly Wind Speed Observations. Journal of Applied Meteorology, 30(6), 823-833, doi:doi:10.1175/15200450(1991)030<0823:TAOHWS>2.0.CO;2.

Brockwell, P. J., \& Marquardt, T. (2005). Levy-driven and fractionality integrated ARMA process with continuous time parameter. Statistica Sinica, 15, 477-494.

Brody C.D., Syroka J., Zervos M. (2002) Dynamical Pricing of Weather Derivatives. Quantitave Finance, 2, 189-198

Brown, B. G., Katz, R. W., \& Murphy, A. H. (1984). Time-series models to simulate and forecast wind speed and wind power, . Journal of Climate and Applied Meteorology, 23, 1184-1195.

Cao, M., and Wei, J (2003) Weather derivatives: A new class of financial instruments. Working Paper, University of Toronto, Toronto.

Cao M, Wei J (2004) Weather Derivatives valuation and market price of weather risk. Journal of Future Markets, 24 (1), 1065-1089 
Caporin, M., \& Pres, J. (2010). Modelling and forecasting wind speed intensity for weather risk management. Computational Statistics \& Data Analysis, doi:doi:10.1016/j.csda.2010.06.019.

Castino, F., Festa, R., \& Ratto, C. F. (1998). Stochastic modelling of wind velocities time-series. Journal of Wind Engineering and Industrial Aerodynamics, 74-76, 141-151.

Celik, A. N. (2004). A statistical analysis of wind power density based on the Weibull and Rayleigh models at the southern region of Turkey. Renewable Energy, 29(4), 593-604, doi:10.1016/j.renene.2003.07.002.

Challis, S. (1999). Bright Forecast for Profits. Reactions, June edition.

CME (2005). An Introduction to CME Weather Products. http://www.cme.com/edu/res/bro/cmeweather. Accessed January 2007.

Cripps, E., Nott, D., Dunsmuir, W. T. M., \& Wikle, C. (2005). Space-Time Modelling of Sydney Harbour Winds. Australian \& New Zealand Journal of Statistics, 47(1), 3-17, doi:10.1111/j.1467-842X.2005.00368.x.

Daniel, A. R., \& Chen, A. A. (1991). Stochastic simulation and forecasting of hourly average wind speed sequences in Jamaica. Solar Energy, 46(1), 1-11.

Dorfleitner G, Wimmer M (2010) The Pricing of Temperature Futures at The Chicago Mercantile Exchange. Journal of Banking \& Finance. doi:10.1016/j.bankfin.2009.12.004.

Dorvlo, A. S. S. (2002). Estimating wind speed distribution. Energy Conversion and Management, 43(17), 2311-2318, doi:Doi: 10.1016/s0196-8904(01)00182-0.

Garcia, A., Torres, J. L., Prieto, E., \& de Francisco, A. (1998). Fitting wind speed distributions: a case study. Solar Energy, 62(2), 139-144, doi:Doi: 10.1016/s0038-092x(97)00116-3.

Hanley, M. (1999). Hedging the Force of Nature. Risk Professional, 1, 21-25.

Härdle, W. K., and Cabrera, B. L. (2011) The Implied Market Price of Weather Risk. Applied Mathematical Finance, 19(1), 59-95.

Haslett, J., \& Raftery, A. E. (1989). Space-Time Modelling with Long-Memory Dependence: Assessing Ireland's Wind Power Resource. Journal of the Royal Statistical Society. Series C (Applied Statistics), 38(1), 1-50.

Huang, H.-H., Shiu, Y.-M., and Lin, P.-S. (2008) HDD and CDD option pricing with market price of weather risk for Taiwan. The Journal of Futures Markets, 28(8), 790-814.

Huang, Z., \& Chalabi, Z. S. (1995). Use of time-series analysis to model and forecast wind speed. Journal of Wind Engineering and Industrial Aerodynamics, 56, 311-322.

Hull, C.J. (2003) Option, Futures and Other Derivatives. Fifth edn. Prentice Hall, New Jersey

Jaramillo, O. A., \& Borja, M. A. (2004). Wind speed analysis in La Ventosa, Mexico: a bimodal probability distribution case. Renewable Energy, 29(10), 1613-1630, doi:DOI: 10.1016/j.renene.2004.02.001.

Jewson, S., Brix, A., \& Ziehmann, C. (2005). Weather Derivative Valuation: The Meteorological, Statistical, Financial and Mathematical Foundations. Cambridge, UK: Campbridge University Press.

Justus, C. G., Hargraves, W. R., Mikhail, A., \& Graber, D. (1978). Methods for estimating wind speed frequency distributions. Journal Name: J. Appl. Meteorol.; (United States); Journal Volume: 17:3, Medium: X; Size: Pages: 350-385.

Kamal, L., \& Jafri, Y. Z. (1997). Time-series models to simulate and forecast hourly averaged wind speed in Quetta, Pakistan. Solar Energy, 61(1), 23-32.

Kavak Akpinar, E., \& Akpinar, S. (2005). A statistical analysis of wind speed data used in installation of wind energy conversion systems. Energy Conversion and Management, 46(4), 515-532, doi:DOI: 10.1016/j.enconman.2004.05.002.

Kavasseri, R. G., \& Seetharaman, K. (2009). Day-ahead wind speed forecasting using f-ARIMA models. Renewable Energy, 34(5), 1388-1393, doi:DOI: 10.1016/j.renene.2008.09.006.

Lei, M., Shiyan, L., Chuanwen, J., Hongling, L., \& Yan, Z. (2009). A review on the forecasting of wind speed and generated power. Renewable and Sustainable Energy Reviews, 13(4), 915 920, doi:DOI: 10.1016/j.rser.2008.02.002.

Lucas R. (1978) Asset Prices in an Exchange Economy. Econometrica, 46, 1429-1445.

Martin, M., Cremades, L. V., \& Santabarbara, J. M. (1999). Analysis and modelling of time-series of surface wind speed and direction. International Journal of Climatology, 19, 197-209.

Mohandes, M. A., Halawani, T. O., Rehman, S., \& Hussain, A. A. (2004). Support vector machines for wind speed prediction. Renewable Energy, 29(6), 939-947, doi:DOI: 10.1016/j.renene.2003.11.009.

Mohandes, M. A., Rehman, S., \& Halawani, T. O. (1998). A neural networks approach for wind speed prediction. Renewable Energy, 13(3), 345-354, doi:Doi: 10.1016/s0960-1481(98)00001-9. 
Moody, J. E., \& Utans, J. (1992). Principled Architecture Selection for Neural Networks: Applications to Corporate Bond Rating Prediction. In A. P. Refenes (Ed.), Neural Networks in the Capital Markets: John Wiley \& Sons.

More, A., \& Deo, M. C. (2003). Forecasting wind with neural networks. Marine Structures, 16, 35-49.

Nfaoui, H., Buret, J., \& Sayigh, A. A. M. (1996). Stochastic simulation of hourly average wind speed sequences in Tangiers (Morocco). Solar Energy, 56(3), 301-314, doi:Doi: 10.1016/0038092x(95)00103-x.

Nielsen, T. S., Madsen, H., Nielsen, H. A., Pinson, P., Kariniotakis, G., Siebert, N., et al. (2006). Shortterm Wind Power Forecasting Using Advanced Statistical Methods. Paper presented at the The European Wind Energy Conference, EWEC, Athens, Greece,

Pinson, P., \& Kariniotakis, G. N. Wind power forecasting using fuzzy neural networks enhanced with on-line prediction risk assessment. In Power Tech Conference Proceedings, 2003 IEEE Bologna, 23-26 June 20032003 (Vol. 2, pp. 8 pp. Vol.2)

Rehman, S., \& Halawani, T. O. (1994). Statistical characteristics of wind in Saudi Arabia. Renewable Energy, 4(8), 949-956, doi:Doi: 10.1016/0960-1481(94)90229-1.

Richards T, J., Manfredo M, R., Sanders D, R. (2004) Pricing Weather Derivatives. American Journal of Agricultural Economics, 4(86), 1005-1017.

Sfetsos, A. (2000). A comaprison of various forecasting techniques applied to mean hourly wind speed time-series. Renewable Energy, 21, 23-35.

Sfetsos, A. (2002). A novel approach for the forecasting of mean hourly wind speed time series. Renewable Energy, 27, 163-174.

Tol, R. S. J. (1997). Autoregressive Conditional Heteroscedasticity in Daily Wind Speed Measurements. Theoretical and Applied Climatology, 56, 113-122.

Tolman, H. L., \& Booij, N. (1998). Modeling wind waves using wavenumber-direction spectra and a variable wavenumber grid. The Global Atmosphere and Ocean System, 6, 295-309.

Torres, J. L., Garcia, A., De Blas, M., \& De Francisco, A. (2005). Forecast of hourly average wind speed with ARMA models in Navarre (Spain). Solar Energy, 79, 65-77.

Tuller, S. E., \& Brett, A. C. (1984). The Characteristics of Wind Velocity that Favor the Fitting of a Weibull Distribution in Wind Speed Analysis. Journal of Climate and Applied Meteorology, 23(1), 124-134.

Turvey C, G. (2005) The Pricing of Degree-Day Weather Options. Agricultural Finance Review, Spring 2005, 59-85

Wilks DS (2011) Statistical Methods in the Atmospheric Sciences, vol 100. International Geophysics Series, 3rd edn. Academic Press, Oxford, UK.

WRMA (2010). Weather derivatives volume plummets. www.wrma.org/pdf/weatherderivativesvolumeplummets.pdf. Accessed January 2010.

Xu, W., Odening, M., \& Musshof, O. (2008). Indifference Pricing of Weather Derivatives. American Journal of Agricultural Economics, 90(4), 979-993.

Yamada, Y. Simultaneous optimization for wind derivatives based on prediction errors. In Americal Control Conference, Washington, USA, June 11-13, 2008 (pp. 350-355)

Zapranis, A., \& Alexandridis, A. (2008). Modelling Temperature Time Dependent Speed of Mean Reversion in the Context of Weather Derivetive Pricing. Applied Mathematical Finance, 15(4), 355 - 386.

Zapranis, A., \& Alexandridis, A. (2009). Weather Derivatives Pricing: Modelling the Seasonal Residuals Variance of an Ornstein-Uhlenbeck Temperature Process With Neural Networks. Neurocomputing, 73, 37-48.

Zapranis, A., \& Alexandridis, A. (2010). Modeling and forecasting cumulative average temperature and heating degree day indices for weather derivative pricing. Neural Computing \&amp; Applications, 1-15, doi:10.1007/s00521-010-0494-1.

Zapranis, A., \& Refenes, A. P. (1999). Principles of Neural Model Indentification, Selection and Adequacy: With Applications to Financial Econometrics: Springer-Verlag. 
Preprint - Published in Computational Economics, 41 (3), pp. 299-326, 2013.

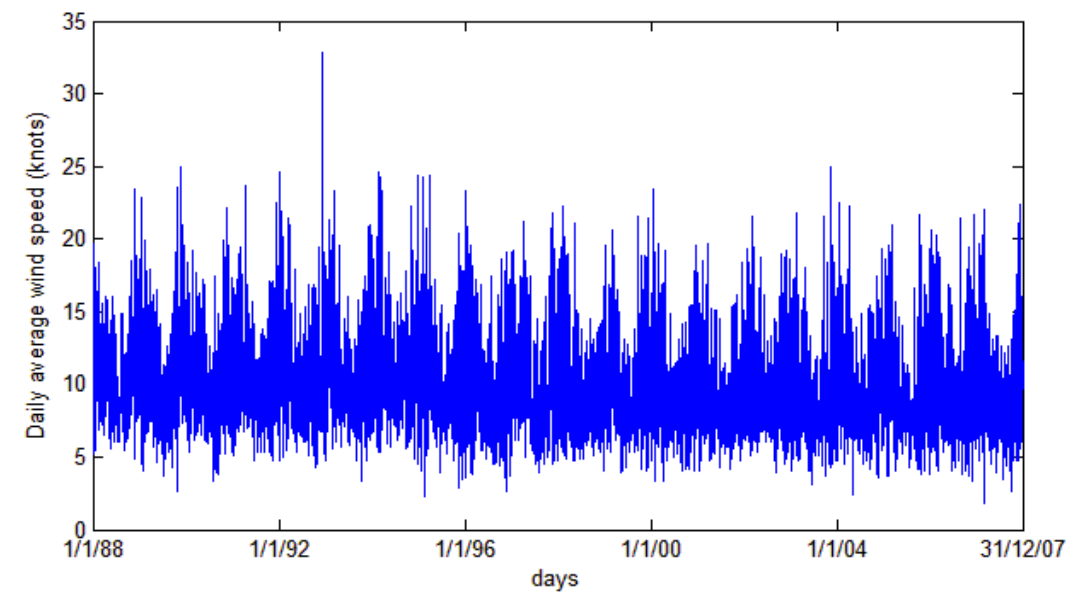

Fig. 1 Daily Average Wind Speed for New York

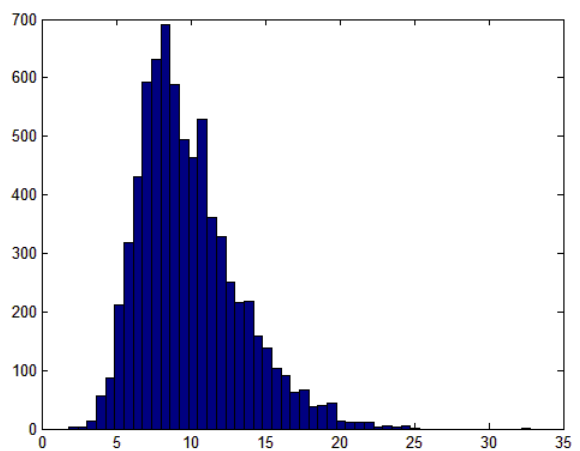

(a)

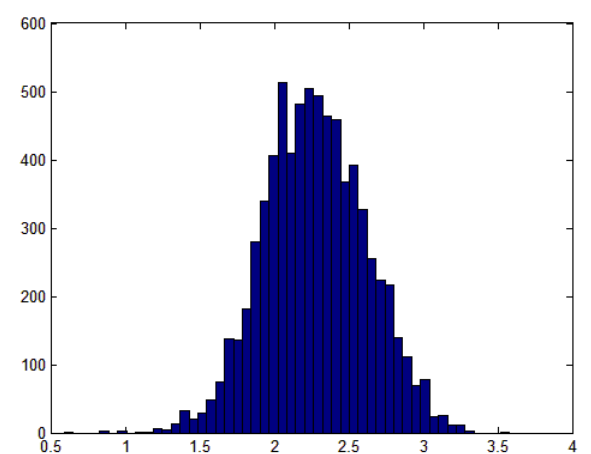

(b)

Fig. 2 Histogram of the (a) original and (b) Box-Cox transformed data

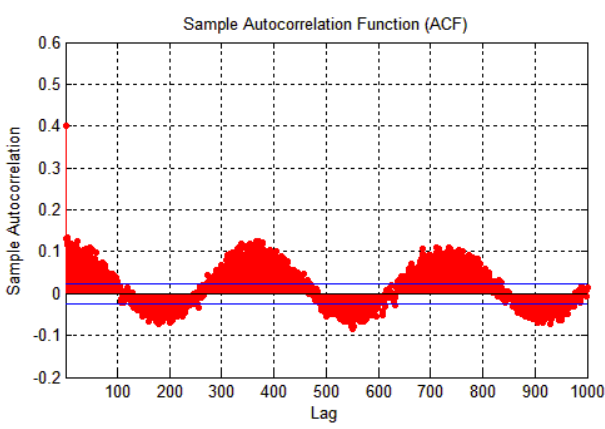

(a)

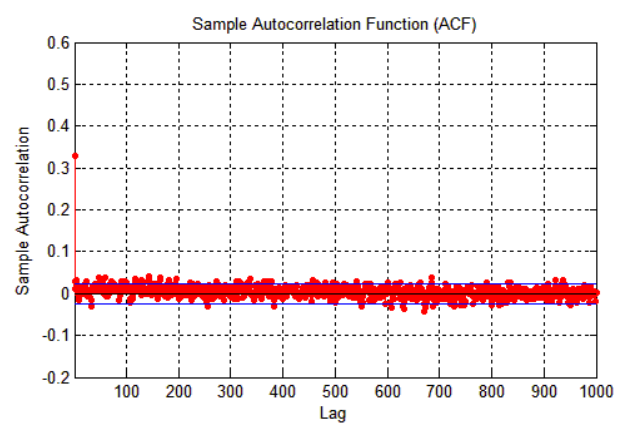

(b)

Fig. 3 The Autocorrelation Function of the transformed DAWSs in New York (a) before and (b) after removing the seasonal mean 
Preprint - Published in Computational Economics, 41 (3), pp. 299-326, 2013.

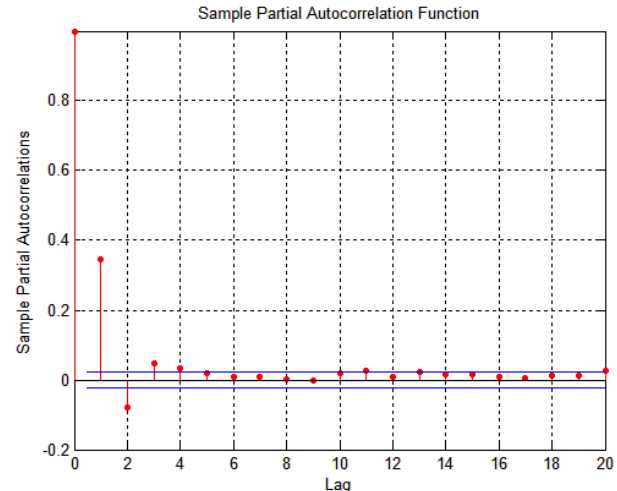

Fig. 4 The Partial Autocorrelation Function of the de-trended and de-seasonalized DAWS in New York

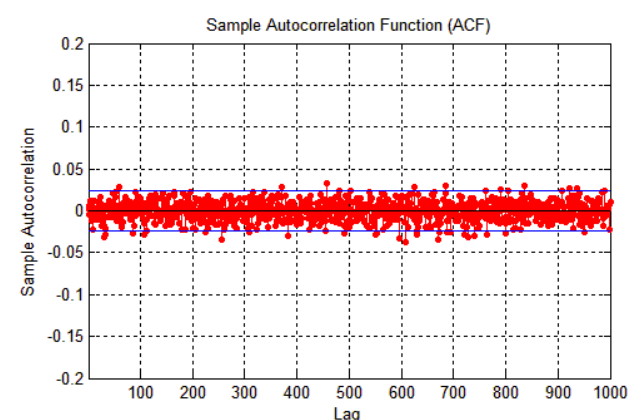

(a)

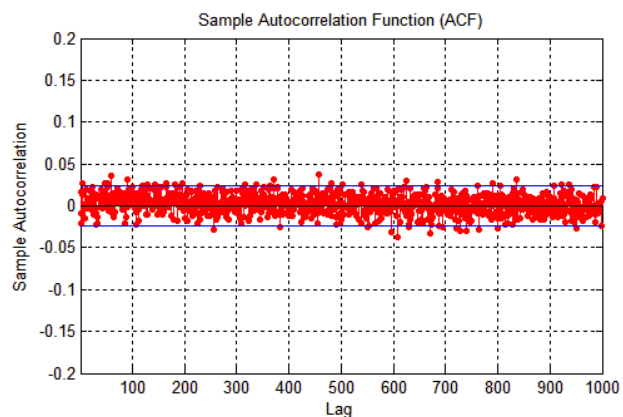

(b)

Fig. 5 Autocorrelation function of the residuals of (a) the linear model and (b) the WN

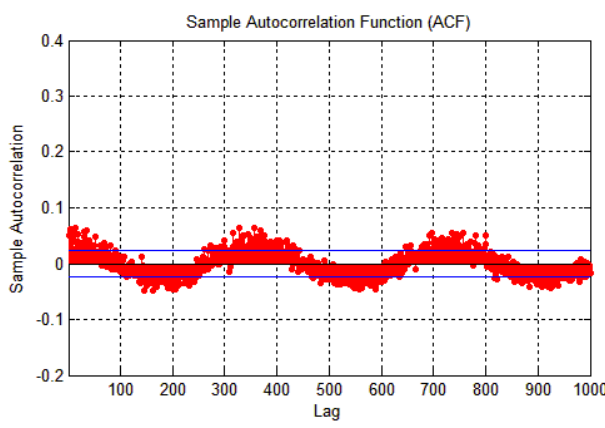

(a)

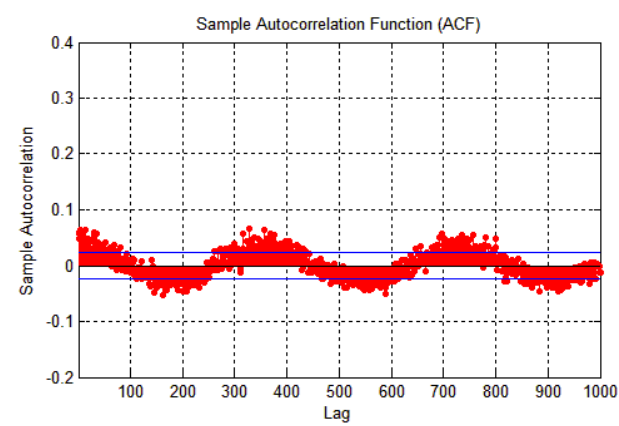

(b)

Fig. 6 Autocorrelation function of the squared residuals of (a) the linear model and (b) the WN

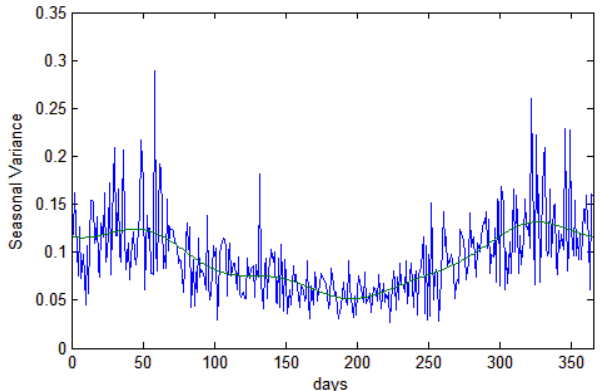

(a)

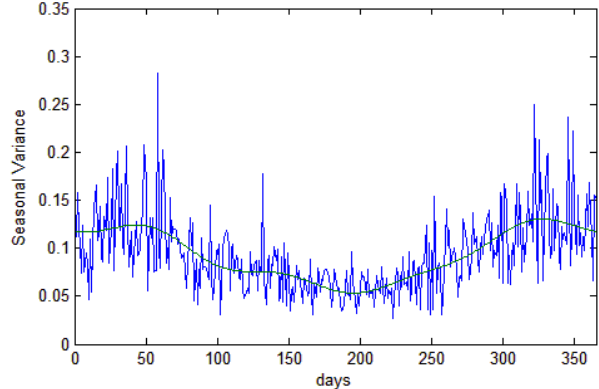

(b)

Fig. 7 Empirical and fitted seasonal variance of (a) the linear model and (b) the WN 
Preprint - Published in Computational Economics, 41 (3), pp. 299-326, 2013.

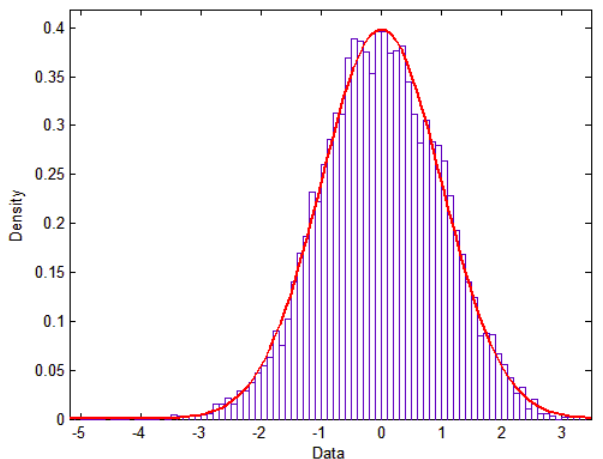

(a)

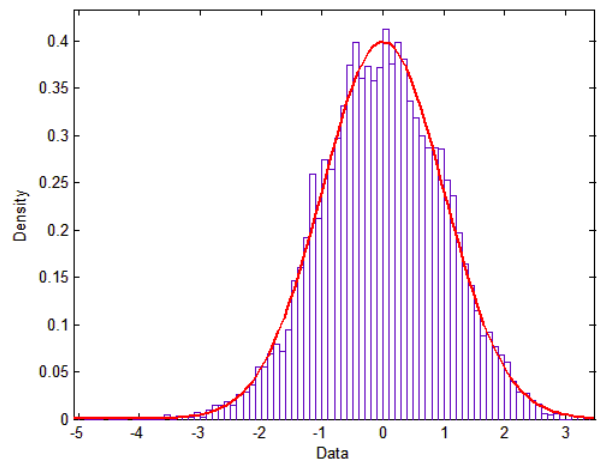

(b)

Fig. 8 Empirical and fitted Normal distribution of the final residuals of (a) the linear model and (b) the WN

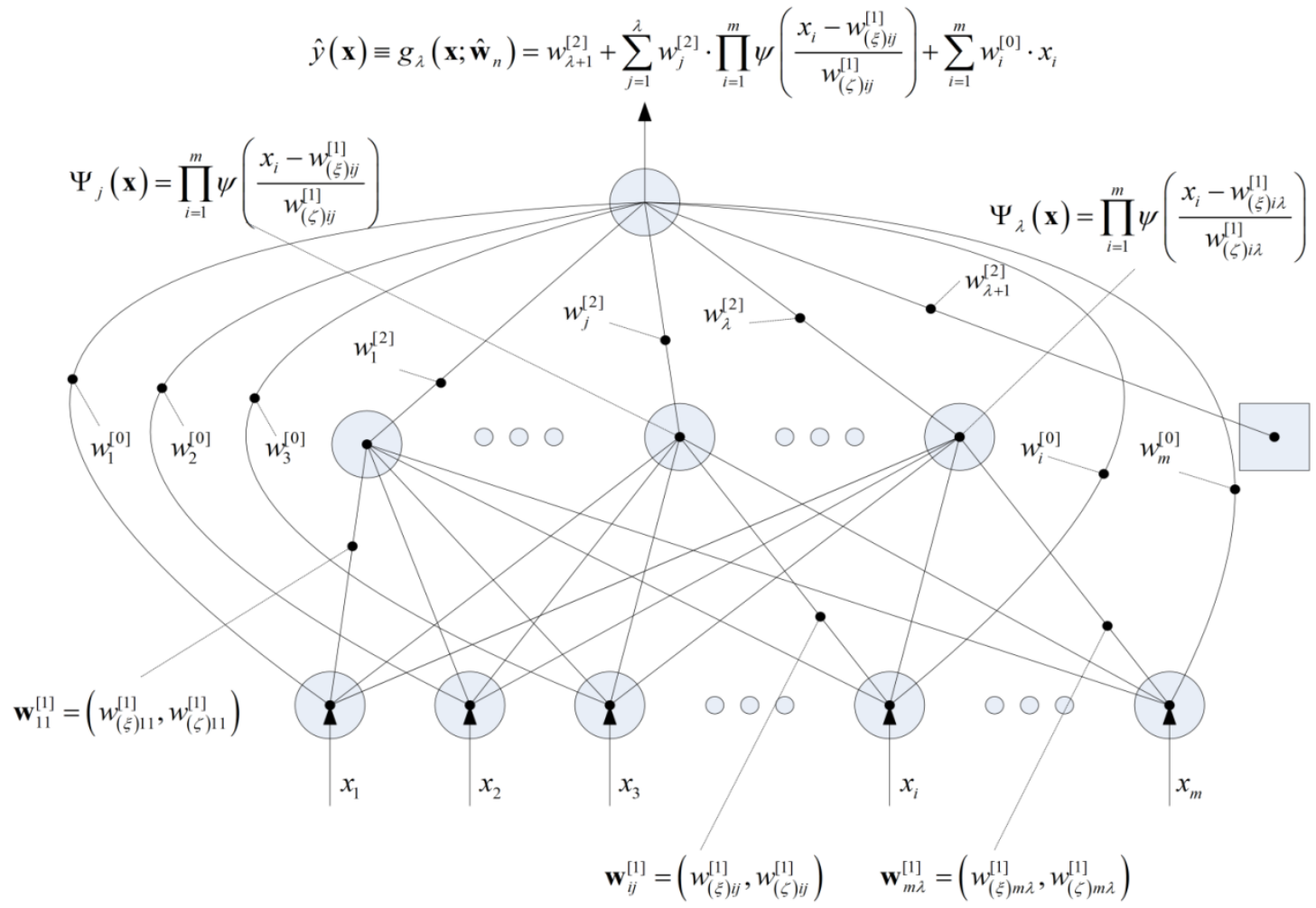

Fig. 9 Structure of a Wavelet Network 
Preprint - Published in Computational Economics, 41 (3), pp. 299-326, 2013.

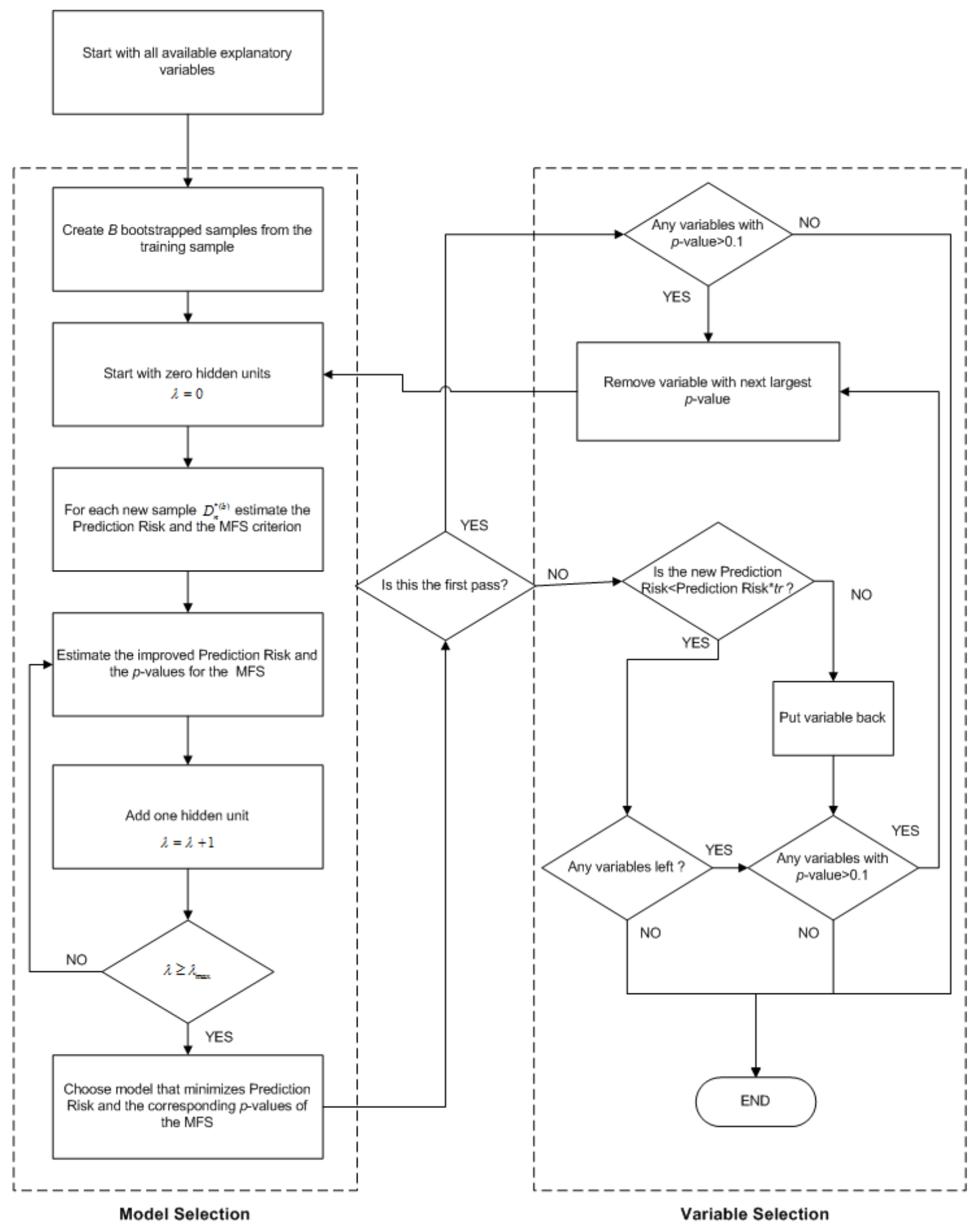

Fig. 10 Model and Variable Selection Algorith 
Preprint - Published in Computational Economics, 41 (3), pp. 299-326, 2013.

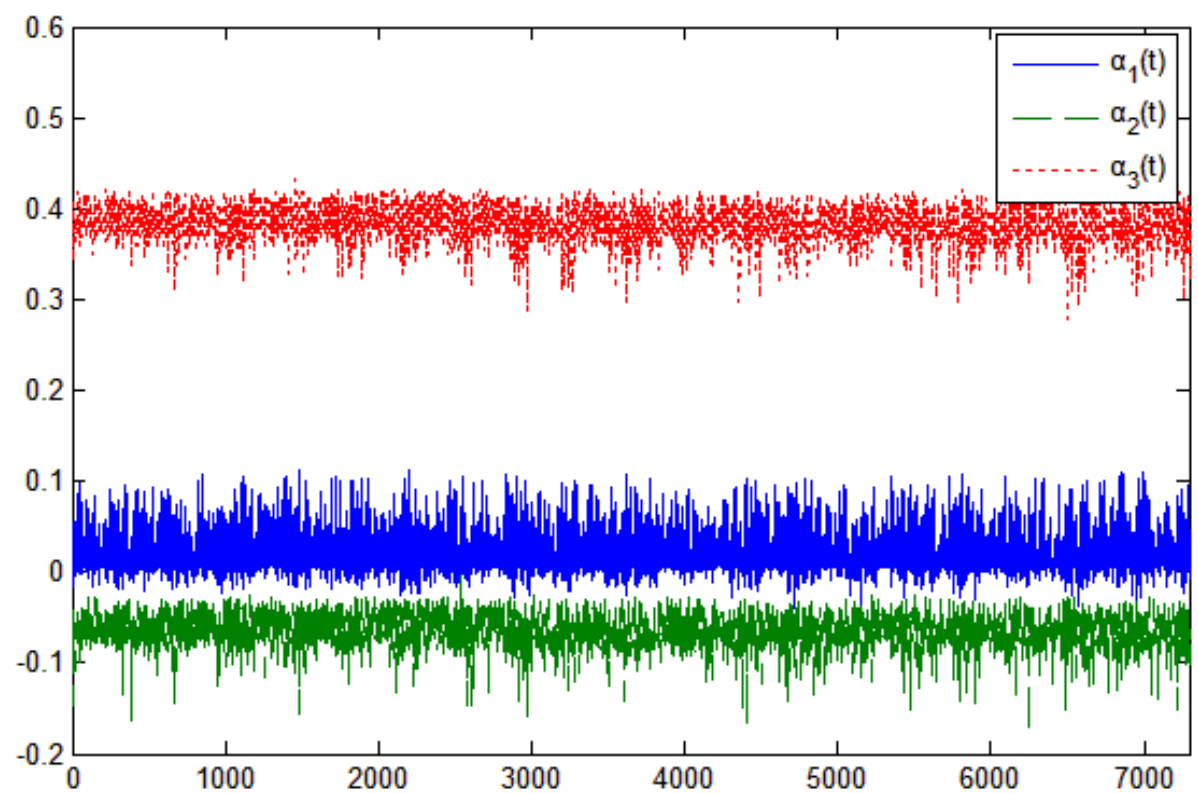

Fig. 11 Daily variations of the speed of mean reversion function $a_{i}(t)$ in New York

Table 1. Descriptive statistics of the wind speed in New York.

\begin{tabular}{llllllllrl}
\hline & Mean & Median & Max & Min & StdDev. & Skew & Kurt & J-B & $p$-value \\
\hline Original & 9.91 & 9.3 & 32.8 & 1.8 & 3.38 & 0.96 & 4.24 & 1595.41 & 0 \\
Transformed & 2.28 & 2.3 & 3.6 & 0.6 & 0.34 & 0.00 & 3.04 & 0.51 & 1 \\
\hline
\end{tabular}

$\mathrm{J}-\mathrm{B}=\mathrm{Jarque}-\mathrm{Bera}$ statistic

$p$-value $=p$-values of the $\mathrm{J}-\mathrm{B}$ statistic

Table 2. Estimated parameters of the seasonal component.

\begin{tabular}{cccccc}
\hline$a_{0}$ & $b_{0}$ & $a_{1}$ & $f_{1}$ & $b_{1}$ & $g_{1}$ \\
\hline 2.3632 & -0.000024 & 0.0144 & 827.81 & 0.1537 & 28.9350 \\
\hline
\end{tabular}

Table 3. Estimated parameters of the linear AR(4) model.

\begin{tabular}{lcccc}
\hline Parameter & $\mathrm{AR}(1)$ & $\mathrm{AR}(2)$ & $\mathrm{AR}(3)$ & $\mathrm{AR}(4)$ \\
\hline Value & 0.3617 & -0.0999 & 0.0274 & 0.0216 \\
$p$-value & 0.0000 & 0.0000 & 0.0279 & 0.0657 \\
\hline
\end{tabular}

Table 4. Estimated parameters of the seasonal variance in the case of the linear model.

\begin{tabular}{ccccccccc}
\hline$c_{0}$ & $c_{1}$ & $c_{2}$ & $c_{3}$ & $c_{4}$ & $d_{1}$ & $d_{2}$ & $d_{3}$ & $d_{4}$ \\
\hline 0.0932 & 0.000032 & -0.0041 & 0.0015 & -0.0028 & 0.0358 & -0.0025 & -0.0048 & -0.0054 \\
\hline
\end{tabular}

Table 5. Descriptive statistics of the residuals for the linear AR(4) model.

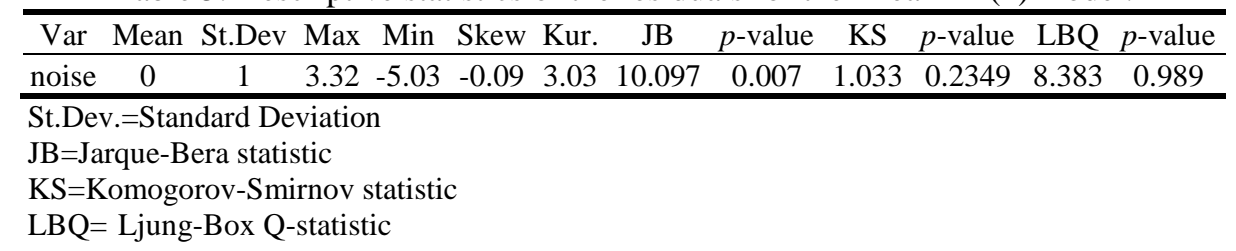


Preprint - Published in Computational Economics, 41 (3), pp. 299-326, 2013.

Table 6. Variable selection with backward elimination in New York.

\begin{tabular}{cccccccc}
\hline $\begin{array}{c}\text { Step } \\
\text { remove (lag) }\end{array}$ & $\begin{array}{c}\text { Variable to } \\
\text { renter (lag) }\end{array}$ & $\begin{array}{c}\text { Variables in } \\
\text { model }\end{array}$ & $\begin{array}{c}\text { Hidden Units } \\
\text { (Parameters) }\end{array}$ & $\begin{array}{c}n / p \\
\text { Ratio }\end{array}$ & $\begin{array}{c}\text { Empirical } \\
\text { Loss }\end{array}$ & $\begin{array}{c}\text { Prediction } \\
\text { Risk }\end{array}$ \\
\hline- & & & 7 & $1(23)$ & 317.4 & 0.0467 & 0.0938 \\
1 & 7 & - & 6 & $1(20)$ & 365.0 & 0.0467 & 0.0940 \\
2 & 5 & - & 5 & $1(17)$ & 429.4 & 0.0467 & 0.0932 \\
3 & 6 & - & 4 & $2(23)$ & 317.4 & 0.0467 & 0.0938 \\
4 & 4 & - & 3 & $2(18)$ & 405.6 & 0.0468 & 0.0937 \\
\hline
\end{tabular}

The algorithm concluded in 4 steps. In each step the following are presented: which variable is removed, the number of hidden units for the particular set of input variables and the parameters used in the wavelet network, the ratio between the parameters and the training patterns, the empirical loss and the prediction risk.

Table 7. Estimated parameters of the seasonal variance in the case of the WN.

\begin{tabular}{ccccccccc}
\hline$c_{0}$ & $c_{1}$ & $c_{2}$ & $c_{3}$ & $c_{4}$ & $d_{1}$ & $d_{2}$ & $d_{3}$ & $d_{4}$ \\
\hline 0.0935 & -0.000020 & -0.0034 & 0.0014 & -0.0026 & 0.0353 & -0.0016 & -.0042 & -0.0052 \\
\hline
\end{tabular}

Table 8. Descriptive statistics of the residuals for the WN model.

\begin{tabular}{lcccccccccccc}
\hline Var & Mean & St.Dev & Max & Min & Skew & Kur. & JB & $p$-value & KS & $p$-value & LBQ & $p$-value \\
\hline noise & 0 & 1 & 3.32 & -4.91 & -0.08 & 3.04 & 8.84 & 0.0043 & 0.927 & 0.3544 & 13.437 & 0.858 \\
\hline St.Dev.=Standard Deviation & & & & & & & & \\
JB=Jarque-Bera statistic & & & & & & & & \\
KS=Komogorov-Smirnov statistic \\
LBQ= Ljung-Box Q-statistic
\end{tabular}

Table 9. Out-of-sample comparison. 1 month.

\begin{tabular}{lrrr}
\hline & PERSISTENT & AR(4) & WN \\
\hline Md. AE & 2.3000 & 2.3363 & 2.0468 \\
ME & -0.0483 & 0.2117 & -0.0485 \\
MAE & 3.3000 & 2.5403 & 2.5151 \\
Max AE & 8.2000 & 7.9160 & 7.7019 \\
SSE & 507.9300 & 326.6666 & 324.3940 \\
RMSE & 4.0478 & 3.2461 & 3.2348 \\
NMSE & 1.5981 & 1.0278 & 1.0206 \\
MSE & 16.3848 & 10.5376 & 10.4643 \\
MAPE & 0.3456 & 0.2724 & 0.2680 \\
SMAPE & 0.3233 & 0.2555 & 0.2518 \\
POCID & $47 \%$ & $80 \%$ & $80 \%$ \\
IPOCID & $33 \%$ & $33 \%$ & $37 \%$ \\
POS & $100 \%$ & $100 \%$ & $100 \%$ \\
\hline Md. AE-Med & Absoly
\end{tabular}

Md. AE=Median Absolute Error

$\mathrm{ME}=$ Mean Error

MAE $=$ Mean Absolute Error

Max AE=Maximum Absolute Error

$\mathrm{SSE}=$ Sum of Squared Errors

RMSE=Root Mean Square Error

NMSE=Normalized Mean Square Error

$\mathrm{MSE}=$ Mean Square Error

MAPE $=$ Mean Absolute Percentage Error

SMAPE=Symmetric MAPE

POCID $=$ Position of change in direction

IPOCID=Independent POCID

POS=Position of Sign 
Preprint - Published in Computational Economics, 41 (3), pp. 299-326, 2013.

Table 10. Out-of-sample comparison. 2 months.

\begin{tabular}{lrrr}
\hline & PERSISTENT & AR(4) & WN \\
\hline Md.AE & 2.4000 & 2.6393 & 2.6745 \\
ME & 0.1101 & 0.3570 & 0.1616 \\
MAE & 3.3678 & 2.8908 & 2.7967 \\
Max AE & 11.2000 & 10.0054 & 8.3488 \\
SSE & 1054.3500 & 754.9589 & 688.2363 \\
RMSE & 4.2273 & 3.5771 & 3.4154 \\
NMSE & 1.4110 & 1.0103 & 0.9210 \\
MSE & 17.8703 & 12.7959 & 11.6650 \\
MAPE & 0.3611 & 0.3126 & 0.3056 \\
SMAPE & 0.3289 & 0.2808 & 0.2778 \\
POCID & $45 \%$ & $69 \%$ & $69 \%$ \\
IPOCID & $36 \%$ & $38 \%$ & $43 \%$ \\
POS & $100 \%$ & $100 \%$ & $100 \%$ \\
\hline
\end{tabular}

Md. AE=Median Absolute Error

$\mathrm{ME}=$ Mean Error

MAE $=$ Mean Absolute Error

Max AE=Maximum Absolute Error

$\mathrm{SSE}=$ Sum of Squared Errors

RMSE=Root Mean Square Error

NMSE=Normalized Mean Square Error

$\mathrm{MSE}=$ Mean Square Error

MAPE=Mean Absolute Percentage Error

SMAPE=Symmetric MAPE

POCID $=$ Position of change in direction

IPOCID=Independent POCID

POS $=$ Position of Sign

Table 11. Estimation of the Cumulative Rainfall Index for 1 and 2 months using an AR(4) model, WN and historical burn analysis.

\begin{tabular}{ccccc}
\hline & AR(4) & WN & HBA & Actual \\
\hline 1 month & 305.1 & 312.7 & 345.5 & 311.2 \\
2 months & 579.5 & 591.1 & 658.3 & 600.6 \\
\hline
\end{tabular}

